\title{
ON THE UNIFORMIZATION OF SOUSLIN $F$ SETS
}

\author{
R. C. WILLMOTT ${ }^{1}$
}

1. Introduction. A set $E$ in a cross product space $X \times Y$ is said to be uniformized by a set $U$ if $U \subset E$; the projections $\Pi_{X} E$ and $\Pi_{X} U$ of $E$ and $U$ onto $X$ coincide; and for each $x \in \Pi_{X} E$ the set $(\{x\} \times Y) \cap U$ of points of $U$ above $x$ is a singleton. (For a detailed discussion of the problem of uniformization see C. A. Rogers and R. C. Willmott [6].) M. Kondô [2] showed that if $E$ is the complement of a Souslin $\mathcal{F}$ set in the Euclidean plane (or, more generally, the cross product of two complete separable metric spaces), then a uniformizing set $U$ may be chosen which is again the complement of a Souslin $\mathcal{F}$ set. He showed further [2, Theorem 7, p. 230] that if $E$ is a Souslin $F$ set then $U$ may be chosen to be a set from the projective class $B_{2}=P_{2} \cap C_{2}$, where $P_{2}$ is the family of projections of complements of Souslin $\mathcal{F}$ sets and $C_{2}$ is the family of complements of sets of $P_{2}$.

In a recent paper, C. A. Rogers and $R$. C. Willmott [6], studying the first result of Kondô, removed almost all conditions on the space $X$. They also considered the case where $Y$ is a descriptive Borel set (see C.A. Rogers [4]), obtaining a partial uniformization result (Theorem 19) in terms of a complement of a Souslin $\mathcal{F}$ set. M. Sion [8], studying the case of an analytic set (which is a Souslin $\mathcal{F}$ set in a complete separable metric space; see, e.g. Bressler and Sion [1, Theorem 5.7]) also removed almost all conditions on $X$ and some on $Y$, and concentrated his attention on the properties of the uniformizing set as a function, e.g. measurable for a large class of measures, not concerning himself with its set-theoretic properties.

In this paper, using methods of Rogers and Willmott [6], the second result of Kondô is generalized in Theorem 2 by removing all conditions on $X$, and by finding for a Souslin $\mathcal{F}$ set $E$ a uniformizing set $U$ which is the difference between $E$ and a $D_{\sigma}$-set, where $D$ is the family of differences of Souslin $\mathcal{F}$ sets. $U$ is a member of the family of $D_{\sigma \delta}$-sets, a subset of $B_{2}$ in a complete separable metric space. It is known that there exist Souslin $F$ sets in the plane which cannot be uniformized either by a Souslin $\mathcal{F}$ set or by the complement of a Souslin $F$ set (see, e.g. [7, p. 57]). The case where $Y$ is a descriptive Borel set is also considered and a result, Theorem 3 , is obtained for a Souslin $\mathcal{F}$ set using the difference between the Souslin $\mathcal{F}$ set and a

Received by the editors September 6, 1968.

1 This paper was written while the author was a fellow of the 1968 Summer Research Institute of the Canadian Mathematical Congress. 
$D_{\sigma}$-set, which is analogous to that obtained in [6, Theorem 19], for the complement of a Souslin $\mathcal{F}$ set using the complement of a Souslin F set.

I am grateful to Professor C. A. Rogers for an observation which led to a reduction of the type of the uniformizing set in these theorems.

2. Definitions and notation. All definitions and notation are those of [6]. The following should suffice for those parts of this paper which are independent or are statements of theorems.

$I$ denotes the family of sequences of positive integers. For $i \in I$, $i \mid n$ is the $n$-tuple $i_{1}, i_{2}, \cdots, i_{n}$; and

$$
I_{i \mid n}=\{j \in I: j|n=i| n\},
$$

called a Baire interval of order $n$. The Baire intervals form a base for a topology on $I$; with this topology $I$ is a homeomorph of the irrational numbers.

Souslin $\mathcal{F}$ in $X$ is the family of sets $A$ having a representation of the form

$$
A=\bigcup_{i \in I} \bigcap_{n=1}^{\infty} A(i \mid n),
$$

where each set $A(i \mid n)$ is closed in $X$. This Souslin representation is said to be disjoint if for each $i, j \in I$,

$$
\bigcap_{n=1}^{\infty} A(i \mid n) \cap \bigcap_{n=1}^{\infty} A(j \mid n)=\varnothing \quad \text { if } i \neq j .
$$

In a complete separable metric space, Souslin $\mathcal{F}$ coincides with the classical family of analytic sets (see, e.g. Bressler and Sion [1, Theorem 5.7]). Souslin sets with disjoint representations are studied in [6, $\$ \$ 4,5$ and 8$]$.

Borel Souslin $\mathfrak{F}$ in $X$ is the smallest family containing Souslin $\mathfrak{F}$ in $X$ and closed under complementation and countable unions.

A function $K$ from $I$ to the compact subsets of a topological space $X$ is said to be semicontinuous if for each $i_{0} \in I$ and open set $G$ of $X$ with $K\left(i_{0}\right) \subset G$, there exists an open set 0 of $I$ with $I_{0} \in 0$ and $K[0] \subset G$. A set in a Hausdorff space $X$ is called descriptive Borel if it has a representation of the form $K[I]=\bigcup_{i \in I} K(i)$ where $K$ is a semicontinuous function from $I$ to the compact subsets of $X$ such that $K(i) \cap K(j)=\varnothing$ if $i \neq j$. For properties of descriptive Borel sets see C. A. Rogers [4].

3. Theorems. We establish our main result in $X \times I$ and subse- 
quently use mapping techniques of Rogers and Willmott [6] to extend it to other spaces.

Let $D$ be the family of differences of Souslin $F$ sets. Then the uniformizing set in the following theorem

$$
U=A \sim \bigcup_{n=1}^{\infty} W_{n}=\bigcap_{n=1}^{\infty}\left(A \sim W_{n}\right)
$$

is an $\mathcal{E}_{\delta}$-set, where $\mathcal{E}$ is the family of sets $E$ of the form

$$
E=A \sim(B \sim C)=(A \sim B) \cup(A \cap B \cap C),
$$

where $A, B$ and $C$ are Souslin $\mathcal{F}$ sets. Clearly

$$
\mathcal{E}_{\delta} \subset D_{\sigma \delta} \subset \text { Borel Souslin } F \text {. }
$$

In a complete separable metric space, $B_{2}$ contains Souslin $\mathcal{F}$, is closed under complementation and countable unions (see, e.g. $[7$, p. 21]) and hence contains Borel Souslin $\mathcal{F}$.

Theorem 1. Let $X$ be a topological space. Then a Souslin $F$ set $A$ in $X \times I$ can be uniformized by the difference between $A$ and a $D_{\sigma}$-set.

Proof. Let $A$ be a Souslin $\mathfrak{F}$ set in $X \times I$. Then, by the first part of the proof of Theorem 17 in [6], $A$ has a representation

$$
A=\bigcup_{i \in I} \bigcap_{n=1}^{\infty} F(i \mid n)
$$

where for each $i \in I$ and $n \geqq 1$,

$$
F(i \mid n)=B(i \mid n) \times C(i \mid n)
$$

where $B(i \mid n)$ is closed in $X$ and $C(i \mid n)$ is empty or is a Baire interval in $I$ of order $\geqq n$. We may suppose that for each $i \in I$ and $n \geqq 1$, $C(i \mid n+1) \subset C(i \mid n)$, for otherwise, setting

$$
F^{\prime}(i \mid n)=B(i \mid n) \times \bigcap_{m=1}^{n} C(i \mid m),
$$

we have the $F^{\prime}(i \mid n)$ of the required form since two Baire intervals are either disjoint or one is contained in the other, and clearly

$$
\bigcup_{i \in I} \bigcap_{n=1}^{\infty} F^{\prime}(i \mid n)=\bigcup_{i \in I} \bigcap_{n=1}^{\infty} F(i \mid n)=A \text {. }
$$

Now for $i \in I, m \geqq 1$, set

$$
A(i \mid m)=\bigcup_{j \in I_{i \mid m}} \bigcap_{n=1}^{\infty} F(j \mid n) \subset A \cap F(i \mid m) .
$$


Let

$$
\begin{aligned}
& V_{1}=\bigcup_{n_{1}=2}^{\infty}\left[A\left(n_{1}\right) \cap\left(\bigcup_{p=1}^{n_{1}-1} \Pi_{X} A(p) \times I\right)\right.\left.\sim \bigcup_{p=1}^{n_{1}-1} A(p)\right], \\
& V_{2}=\bigcup_{n_{1}=1}^{\infty} \bigcup_{n_{2}=2}^{\infty}\left[A\left(n_{1}, n_{2}\right) \cap\left(\bigcup_{p=1}^{n_{2}-1} \Pi_{X} A\left(n_{1}, p\right) \times I\right)\right. \\
&\left.\sim\left(\bigcup_{p=1}^{n_{2}-1} A\left(n_{1}, p\right) \cup \bigcup_{p=1}^{n_{1}-1} A(p)\right)\right],
\end{aligned}
$$

and, in general,

$$
\begin{aligned}
V_{s}= & \bigcup_{n_{1}=1}^{\infty} \ldots \bigcup_{n_{s-1}=1}^{\infty} \bigcup_{n_{s}=2}^{\infty} \\
& \cdot\left[A\left(n_{1}, \cdots, n_{s}\right) \cap\left(\bigcup_{p=1}^{n_{s}-1} \Pi_{X} A\left(n_{1}, \cdots, n_{s-1}, p\right) \times I\right)\right. \\
& \sim\left(\bigcup_{p=1}^{n_{s}-1} A\left(n_{1}, \cdots, n_{s-1}, p\right) \cup \bigcup_{p=1}^{n_{s-1}-1} A\left(n_{1}, \cdots, n_{s-2}, p\right)\right. \\
& \left.\left.\cdots \cup \bigcup_{p=1}^{n_{1}-1} A(p)\right)\right] .
\end{aligned}
$$

Finally, set $U=A \sim \bigcup_{s=1}^{\infty} V_{s}$ and check:

(1) As $I_{i \mid m}$ is homeomorphic to $I$, each $A(i \mid m)$ is a Souslin $\mathcal{F}$ set in $X \times I$, and so, by the result in [5], $\Pi_{X} A(i \mid m)$ is Souslin $\mathcal{F}$ in $X$, as is $\Pi_{X} A(i \mid m) \times I$ in $X \times I$. Hence each $V_{s}$ is a $D_{\sigma}$-set, as is $\bigcup_{s=1}^{\infty} V_{s}$.

(2) $U \subset A$ trivially.

(3) $\Pi_{X} A=\Pi_{X} U$. By (2) it suffices to show that $\Pi_{X} A \subset \Pi_{X} U$. Suppose $x \in \Pi_{X} A$. As $A=\bigcup_{p=1}^{\infty} A(p)$, there exists a least integer, $i_{1}$, such that $x \in \Pi_{X} A\left(i_{1}\right)$.

By construction of $V_{1}$ and the choice of $i_{1}, V_{1}$ contains all points of $A$ outside $A\left(i_{1}\right)$ which project onto $x$, but contains no points of $A\left(i_{1}\right)$ which project onto $x$. That is

$$
\Pi_{X}^{-1}(x) \cap\left(A \sim V_{1}\right)=\Pi_{X}^{-1}(x) \cap A\left(i_{1}\right) \neq \varnothing .
$$

As $A\left(i_{1}\right) \subset F\left(i_{1}\right)=B\left(i_{1}\right) \times C\left(i_{1}\right)$, we have

$$
\Pi_{X}^{-1}(x) \cap A\left(i_{1}\right) \subset\{x\} \times C\left(i_{1}\right),
$$

and $x \in B\left(i_{1}\right), C\left(i_{1}\right) \neq \varnothing$.

Again, $A\left(i_{1}\right)=\bigcup_{p=1}^{\infty} A\left(i_{1}, p\right)$, and there exists a first integer, $i_{2}$, such that $x \in \Pi_{x} A\left(i_{1}, i_{2}\right)$. By construction of $V_{2}$ and the choice of $i_{1}$ and $i_{2}, V_{2}$ contains all points of $A\left(i_{1}\right)$ outside $A\left(i_{1}, i_{2}\right)$ which project 
onto $x$, but contains no points of $A\left(i_{1}, i_{2}\right)$ which project onto $x$. Then $V_{1} \cup V_{2}$ contains all points of $A$ outside $A\left(i_{1}, i_{2}\right)$ which project onto $x$, but contains no points of $A\left(i_{1}, i_{2}\right)$ which project onto $x$, i.e.

$$
\Pi_{X}^{-1}(x) \cap\left[A \sim\left(V_{1} \cup V_{2}\right)\right]=\Pi_{X}^{-1}(x) \cap A\left(i_{1}, i_{2}\right) \neq \varnothing .
$$

Again $\Pi_{\bar{X}}^{-1}(x) \cap A\left(i_{1}, i_{2}\right) \subset\{x\} \times C\left(i_{1}, i_{2}\right), x \in B\left(i_{1}, i_{2}\right)$, and $C\left(i_{1}, i_{2}\right) \neq \varnothing$. Continuing, we obtain $i \in I$ such that for each $n \geqq 1$,

$$
\begin{aligned}
& \Pi_{X}^{-1}(x) \cap\left[A \sim\left(\bigcup_{s=1}^{n} V_{s}\right)\right]=\Pi_{X}^{-1}(x) \cap A(i \mid n) \neq \varnothing, \\
& \Pi_{X}^{-1}(x) \cap A(i \mid n) \subset\{x\} \times C(i \mid n),
\end{aligned}
$$

$x \in B(i \mid n)$, and $C(i \mid n) \neq \varnothing$.

Now, as $C(i \mid n+1) \subset C(i \mid n)$, and as $C(i \mid n)$ is a Baire interval of order $\geqq n$, it follows that $\bigcap_{n=1}^{\infty} C(i \mid n)$ is a singleton. Let $\bigcap_{n=1}^{\infty} C(i \mid n)$ $=\{y\}$. Then

$$
(x, y) \in \bigcap_{n=1}^{\infty}[B(i \mid n) \times C(i \mid n)]=\bigcap_{n=1}^{\infty} F(i \mid n)=\bigcap_{n=1}^{\infty} A(i \mid n) .
$$

Then for all $n$,

$$
(x, y) \in \Pi_{X}^{-1}(x) \cap A(i \mid n)=\Pi_{X}^{-1}(x) \cap\left[A \sim \bigcup_{s=1}^{n} V_{*}\right]
$$

and so

$$
(x, y) \in \bigcap_{n=1}^{\infty}\left(A \sim \bigcup_{s=1}^{n} V_{s}\right)=A \sim \bigcup_{s=1}^{\infty} V_{s}=U .
$$

Hence $x \in \Pi_{X} U$ and $\Pi_{X} A \subset \Pi_{X} U$.

(4) Finally, for $x \in \Pi_{X} A=\Pi_{X} U$, we have from a formula in (3), for each $n$,

$$
\Pi_{X}^{-1}(x) \cap\left[A \sim \bigcup_{i=1}^{n} V_{s}\right] \subset\{x\} \times C(i \mid n)
$$

and hence

$$
\begin{aligned}
\Pi_{X}^{-1}(x) \cap U & =\bigcap_{n=1}^{\infty}\left[\Pi_{X}^{-1}(x) \cap\left(A \sim \bigcup_{8=1}^{n} V_{n}\right)\right] \\
& \subset \bigcap_{n=1}^{\infty}[\{x\} \times C(i \mid n)]=\{x\} \times \bigcap_{n=1}^{\infty} C(i \mid n),
\end{aligned}
$$

so that $\Pi_{\bar{X}}^{-1}(x) \cap U$ is a singleton. This completes the proof. 
The next theorem generalizes Kondô's result on the uniformization of a Souslin $\mathcal{F}$ set in the product of two complete separable metric spaces. It is well known that a complete separable metric space is a continuous one-to-one image of a closed subset of $I$ (see, e.g. [3, p. 443]).

THEOREM 2. Suppose $Y$ is a continuous one-to-one image of a closed subset $H$ of $I, X$ is a topological space, and $E$ is a Souslin $F$ set in $X \times Y$. Then $E$ can be uniformized by the difference between $E$ and $a$ $D_{\sigma}$-set.

Proof. Let $g$ be a continuous function defined on $H$ which maps $H$ one-to-one onto $Y$. Define $\phi: X \times H \rightarrow X \times Y$ by setting

$$
\phi(x, i)=(x, g(i)) \quad \text { for all } x \in X, \quad i \in H .
$$

Then, as in the proof of Theorem 18 in [6], $\phi$ is a continuous one-toone function on $X \times H$ onto $X \times Y$ which preserves Souslin $\mathcal{F}$ sets, i.e. if $B$ is a Souslin $\mathcal{F}$ set in $X \times H$, then $\phi[B]$ is Souslin $\mathcal{F}$ in $X \times Y$. As $\phi$ is continuous, $A=\phi^{-1}[E]$ is a Souslin $\mathcal{F}$ set in $X \times H$ and hence in $X \times I$ as $H$ is closed in $I$. By Theorem 1 , there is a $D_{\sigma}$-set, $V$, in $X \times I$ such that $A \sim V$ uniformizes $A$. We have $V \subset A \subset X \times H$ and evidently $V$ is also a $D_{\sigma}$-set in $X \times H$. Clearly

$$
U=\phi[A \sim V]=\phi[A] \sim \phi[V]=E \sim \phi[V]
$$

uniformizes $E$. Finally, $\phi[V]$ is a $D_{\sigma}$-set in $X \times Y$ since $\phi$ preserves Souslin $\mathcal{F}$ sets and unions, and being one-to-one, also preserves differences.

Theorem 3. Let $X$ be a topological space, $Y$ a Hausdorff space with a representation $Y=K[I]$ as a descriptive Borel set. Suppose that each open set in $X \times Y$ has a disjoint Souslin representation. Let $A$ be a Souslin $\mathcal{F}$ set in $X \times Y$. Then there is a set $U$ which is the difference between $A$ and $a D_{\sigma}$-set in $X \times Y$, and which satisfies

(a) $U \subset A$,

(b) $\Pi_{X} A=\Pi_{X} U$, and

(c) for each $x \in \Pi_{X} A$, the set $\Pi_{Y}\left[\Pi_{\bar{X}}^{-1}(x) \cap U\right]$ is compact and contained in some set $K(i)$ with $i \in I$.

Proof. Let $A$ have the representation

$$
A=A[I], \quad A(i)=\bigcap_{n=1}^{\infty} A(i \mid n),
$$

the sets $A(i \mid n)$ being closed. Then, as in the proof of Theorem 19 in [6], let 


$$
X \times Y=F[I], \quad F(i)=\bigcap_{n=1}^{\infty} F(i \mid n)
$$

be a representation of $X \times Y$ such that the sets $F(i \mid n)$ are closed, and each set $A(i \mid n)$ is the union of those fragments $F(i)$ that it meets, and such that for each $i \in I$, there is a $j \in I$ such that for each integer $m \geqq 1$, there exists an integer $n$ with

$$
F\left[I_{i \mid n}\right] \subset X \times K\left[I_{j \mid m}\right] .
$$

As in the proof of that theorem we define a map $\omega: X \times Y \rightarrow X \times I$ by setting for each $x \in X, y \in Y, \omega(x, y)=(x, i)$, where $i=i(x, y)$ is the unique $i$ in $I$ with $(x, y) \in F(i)$. Then again $\omega A$ is a Souslin $\mathcal{F}$ set in $X \times I$. By Theorem 1 , there exist Souslin $\mathcal{F}$ sets $B_{s}, C_{s}$ in $X \times I$ such that

$$
W=\omega A \sim \bigcup_{8=1}^{\infty}\left(B_{8} \sim C_{8}\right)
$$

uniformizes $\omega A$. Let $U=\omega^{-1}[W]$. Properties (a), (b), and (c) follow for $U$ as in the proof of Theorem 19 in [6]. It remains to show that $U$ is the difference between $A$ and a $D_{\tau}$-set in $X \times Y$. We have

$$
\begin{aligned}
U & =\omega^{-1}\left[\omega A \sim \bigcup_{s=1}^{\infty}\left(B_{s} \sim C_{s}\right)\right] \\
& =\omega^{-1}[\omega A] \sim\left(\bigcup_{s=1}^{\infty}\left(\omega^{-1}\left[B_{s}\right] \sim \omega^{-1}\left[C_{s}\right]\right)\right) .
\end{aligned}
$$

Using Theorem 8 of [6] as in the proof of Theorem 19 in [6], we have $\omega^{-1}\left[B_{8}\right]$ and $\omega^{-1}\left[C_{8}\right]$ Souslin $\mathcal{F}$ sets in $X \times Y$. Finally, by definition of $\omega, \omega^{-1}[\omega F(i)]=F(i)$ for $i$ in $I$, and as each set $A(i \mid n)$ is the union of the fragments $F(i)$ that it meets the same is true of each $A(i)=\bigcap_{n=1}^{\infty} A(i \mid n)$, and so also $\omega^{-1}[\omega A(i)]=A(i)$ for $i$ in $I$. Hence

$$
\begin{gathered}
\omega^{-1}[\omega A]:=\omega^{-1}\left[\omega \bigcup_{i \in I} A(i)\right]=\bigcup_{i \in I} \omega^{-1}[\omega A(i)]=\bigcup_{i \in I} A(i)=A . \\
\text { REFERENCES }
\end{gathered}
$$

1. D. W. Bressler and M. Sion, The current theory of analytic sets, Canad. J. Math. 16 (1964), 207-230.

2. M. Kondô, Sur l'uniformisation des complémentaires analytiques et les ensembles projectifs de la seconde classe, Japan J. Math. 15 (1939), 197-230.

3. K. Kuratowski, Topology, Vol. 1, Academic Press, New York, 1966.

4. C. A. Rogers, Descriptive Borel sets, Proc. Roy. Soc. Ser. A 286 (1965), 455478. 
5. C. A. Rogers and R. C. Willmott, On the projection of Souslin sets, Mathematika 13 (1966), 147-150.

6. - On the uniformization of sets in topological spaces, Acta Math. 120 (1968), 1-52.

7. W. Sierpinski, Les ensembles projectifs et analytiques, Mémor. Sci. Math. No. 112, Gauthier-Villars, Paris, 1950.

8. M. Sion, On uniformization of sets in topological spaces, Trans. Amer. Math. Soc. 96 (1960), 237-245.

UnIVERSitE DE MONTREAL 